\title{
Artificial Intelligence Applications in Tracking Health Behaviors During Disease Epidemics
}

\author{
Kurubaran Ganasegeran and Surajudeen Abiola Abdulrahman
}

\begin{abstract}
The threat of emerging and re-emerging infectious diseases to global population health remains significantly enormous, and the pandemic preparedness capabilities necessary to confront such threats must be of greater potency. Artificial Intelligence (AI) offers new hope in not only effectively pre-empting, preventing and combating the threats of infectious disease epidemics, but also facilitating the understanding of health-seeking behaviors and public emotions during epidemics. From a systems-thinking perspective, and in today's world of seamless boundaries and global interconnectivity, AI offers enormous potential for public health practitioners and policy makers to revolutionize healthcare and population health through focussed, context-specific interventions that promote cost-savings on therapeutic care, expand access to health information and services, and enhance individual responsibility for their health and well-being. This chapter systematically appraises the dawn of AI technology towards empowering population health to combat the rise of infectious disease epidemics.
\end{abstract}

Keywords Artificial intelligence $\cdot$ Health behaviors $\cdot$ Epidemics $\cdot$ Infectious disease $\cdot$ Global health

K. Ganasegeran $(\bowtie)$

Clinical Research Center, Seberang Jaya Hospital, Ministry of Health Malaysia,

Penang, Malaysia

e-mail: medkuru@yahoo.com

\section{S. A. Abdulrahman}

Emergency Medicine Department, James Paget University Hospital,

Great Yarmouth, Norfolk, UK

e-mail: abdulsuraj@gmail.com

D. J. Hemanth (ed.), Human Behaviour Analysis Using Intelligent Systems,

Learning and Analytics in Intelligent Systems 6,

https://doi.org/10.1007/978-3-030-35139-7_7 


\section{Introduction}

Infectious diseases disrespect national and international borders. They pose substantial threats and serious repercussions to global public health security. While the Asia-Pacific region was generally regarded as the main epicenter of emerging infectious diseases, with outbreaks of Avian Flu, Asian Flu and Severe Acute Respiratory Syndrome (SARS) [1], the recent and unexpected emergence of Zika pandemic spurred global concerns about pandemic preparedness capabilities particularly as it relates to training and deployment of healthcare workforce at a massive level, worldwide. Despite coordinated global efforts, containing the "red alert" pandemic of Zika remained a challenge, as both healthcare workers and public health advocates were uncertain about such disastrous contagion causing serious complications including congenital microcephaly in newborns and neurological deficits in adults $[2,3]$.

Control measures were obtunded as public health advocates were initially speculative about the potential transmission route of Zika, while clinicians in hospitals were irresolute, instituting multiple levels of care and management to tackle the complications of Zika. This debacle gave rise to an urgent need to debate the circumstances under which the Zika epidemic has challenged human intelligence behavior and capacity to battle the threat effectively and efficiently.

As population explosions and uncontrolled human mobility across nations catalyzes rapid disease propagation, our next question is, what else above human intelligence could help resolve such unprecedented epidemic crisis? Scientists believe that the time has come to institute analytic technologies - such as Artificial Intelligence (AI) - in healthcare to help prevent and resolve such large disease epidemics $[4,5]$. Adaptive AI applications could mould human behavior to practice preventive behaviors and disease control strategies [6], thereby improving global health. This chapter will systematically discuss the dawn of AI technology in healthcare that could potentially empower the human population to tackle unprecedented infectious disease epidemics.

\section{Artificial Intelligence-The Evolution Begins!}

The human population has witnessed four major revolutions till date (Fig. 1); the foremost being the first industrial revolution that introduced steam engine to the world [7]. This was followed by the second industrial revolution that introduced electrical-energy based productions. The first information revolution was conceptualized during the third industrial revolution in the late 20th century. It was during this time that computers and internet-based knowledge began and has since then shaped human interactions. In early 21 st century, the fourth industrial revolution accelerated the second information revolution. The entire phase of human daily functions transformed with the debut of AI, bringing together massive information 


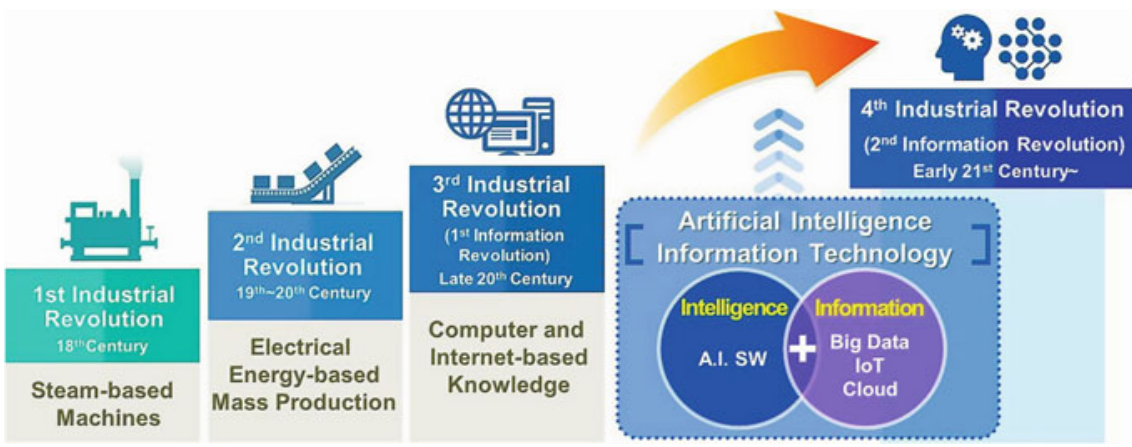

Fig. 1 The four industrial revolutions within the human interface. Source http://cradall.org/ content/being-human-era-fourth-industrial-revolution-and-ai-peter-kearns with original source from worldbank.org. Figure available in public domain with licensee agreement of "fair use," allowing free usage for academic and research purposes

flow from different specialties. These culminated in the rise of Big Data with systems integration across the Internet of Things (IOT) and Cloud Computing Systems.

Current revolutionary era is based on extreme automation for global connectivity, in which AI would definitely play an imperative role as a resource to utilize. At the peak of emergent multi-function contexts of AI and the rise of Big Data Analytics, the United Nations (UN) in 2017 unified global experts to galvanize a dynamic consensus on the adoption and expansion of AI use in delivering good public care services [8]. Succinctly, various stakeholders were assembled together in another UN meeting to assess the role of AI towards achieving Sustainable Developmental Goals (SDGs) [9]. From the healthcare perspective, massive data have been obtained from public health surveillance efforts with the advancement of AI. One major public health field that gained momentum to develop various AI applications for disease prevention was the infectious disease domain [5]. The human population is currently able to access potentially useful massive data sources of infectious disease spread through sentinel reporting systems, national surveillance systems (usually operated by national or regional disease centers such as the Center for Disease Control (CDC)), genome databases, internet search queries (also called infodemiology and infoveillance studies) [10-12], Twitter data analysis [13, 14], outbreak investigation reports, transportation dynamics [15], vaccine reports [16] and human dynamics information [17].

With the influx of massive data volume, effective data integration, management and knowledge extraction systems are required [5]. Epidemic modeling and disease-spread simulations form new horizons to understand the effects of citizen behaviors or government health policy measures [1]. A simple integrated effect of disease knowledge discovery is exhibited in Fig. 2. 


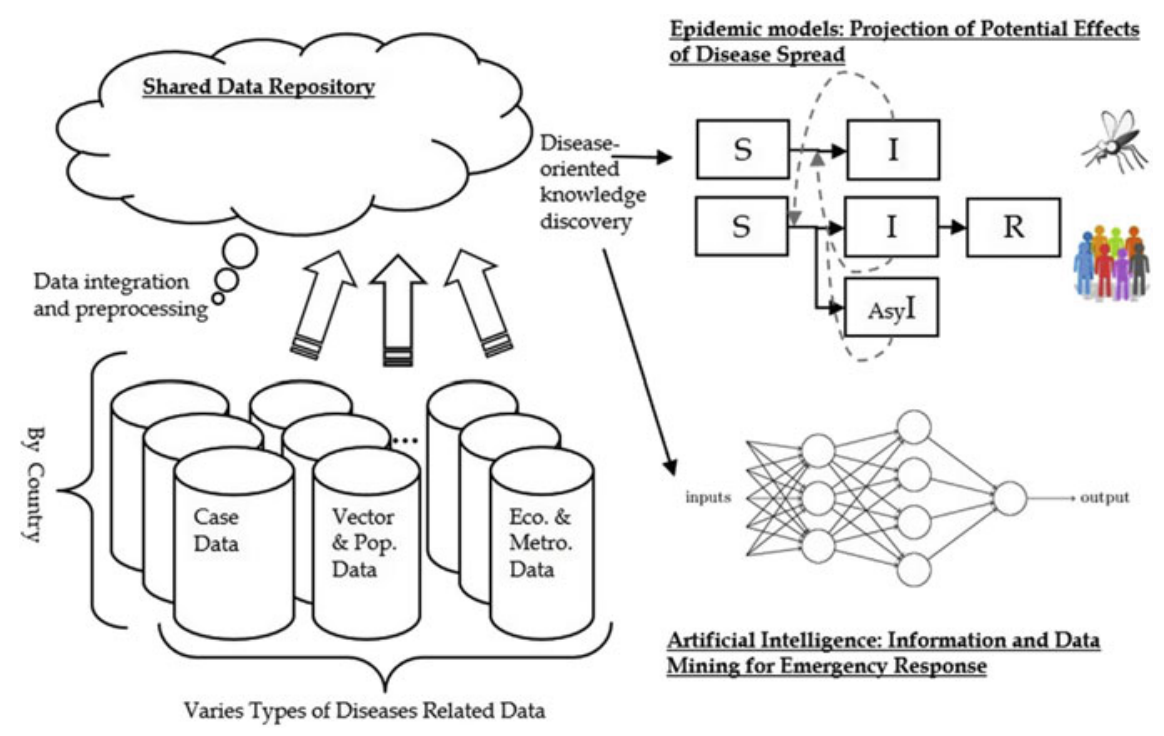

Fig. 2 Integrated conceptual model for infectious diseases using AI. Source Wong et al. [5]

\section{Conceptualizing Artificial Intelligence in Disease Epidemics}

As humans, we are able to perform simple essential tasks such as object detection, visual interpretation and speech recognition.

Our interpretation is instantaneous when we look at an object or image, or when we hear voices or noises surrounding us. Our next question is-could AI perform these essential intellectual tasks as well? The answer is absolutely yes, but in a different mode of function. While human interpretation is solely dependent on cognitive functions, AI requires mathematical algorithms to automate machines for execution of such functions [18]. Machines here refer to programmable computers! An example is to visualize the cause of an outbreak; Dengue, Chikungunya or Zika, of which these diseases are commonly caused by the vector mosquito. In massive epidemics, elimination of the vector is important, and human cognitive functions can never detect all mosquitos in an outbreak investigation area! However, this can be easily detected through deployment of AI in areas which have loads of mosquito vectors to facilitate control measures. Figure 3 exhibits how human and AI technology interpret the vector differently.

While human interpretation is instantaneous, AI evaluates the same image as humans do, but translated into codes [18], facilitating massive detection. While AI aims to mimic human cognitive functions, it lacks intuitive behaviors. Scientists postulate that such synthetic intelligence which could be on par with human intelligence can be called "computational intelligence." However, the primary goal 


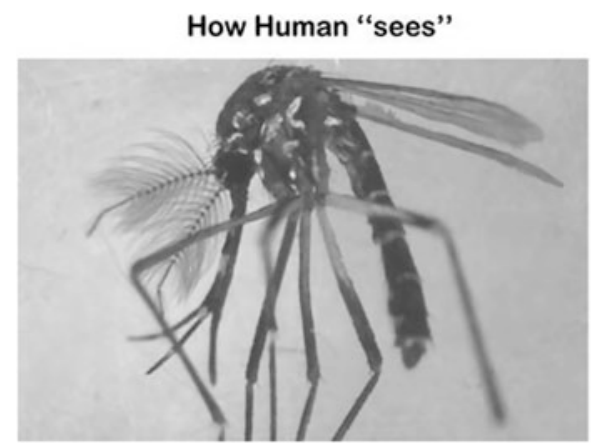

How Computer "sees"
$\left[\begin{array}{lllllllll}1 & 0 & 0 & 0 & 0 & 0 & 1 & 0 & 1 \\ 0 & 1 & 1 & 1 & 1 & 1 & 0 & 0 & 0 \\ 0 & 0 & 1 & 1 & 1 & 1 & 0 & 0 & 0 \\ 1 & 1 & 0 & 0 & 0 & 1 & 1 & 1 & 0 \\ 0 & 1 & 0 & 1 & 0 & 1 & 0 & 0 & 1 \\ 1 & 0 & 1 & 1 & 0 & 1 & 1 & 0 & 1 \\ 0 & 1 & 0 & 0 & 1 & 1 & 0 & 1 & 0 \\ 1 & 0 & 0 & 1 & 1 & 0 & 1 & 1 & 1 \\ 1 & 0 & 1 & 1 & 1 & 1 & 0 & 0 & 0\end{array}\right]$

Fig. 3 Interpreting the vector from the human and AI perspective. Source da Silva Motta et al. [18]

of AI was to create a system programming that is capable to think and act rationally like humans, although such machines may lack intuitive or emotional capabilities. As such, AI has been appropriately defined in simple and straightforward terms, as "a branch of computer science that deals with simulation of intelligent behaviors as humans using computers [19]".

\section{Types of AI}

In principle, there are three types of AI. If a machine is able to think as humans do and perform a task similar to human intellectual capabilities, then that machine functionality is referred to as artificial general intelligence [20]. If a machine performs a single task extremely well, this is known as artificial narrow intelligence [4]. If the same machine out-smart the best humans in all fields from scientific creativity to general wisdom or social skills, this is referred to as artificial super intelligence [4]. At present, virtually all contemporary AI application systems utilize artificial narrow intelligence.

\subsection{Main Subsets of AI}

There are numerous concepts to function underlying AI applications in healthcare. Based on the required functions, these concepts are clumped together to automate a single application - such as tracking infectious disease health seeking behavior. The following sub-sections summarize key concepts of different AI subsets adopted in emerging literature of infectious diseases.

Machine Learning (ML). ML is a subfield of AI that implies learning from previous experiences (Fig. 4). The system finds solution to a problem by extracting 
Fig. 4 Diagram Venn illustrating artificial intelligence and main subsets

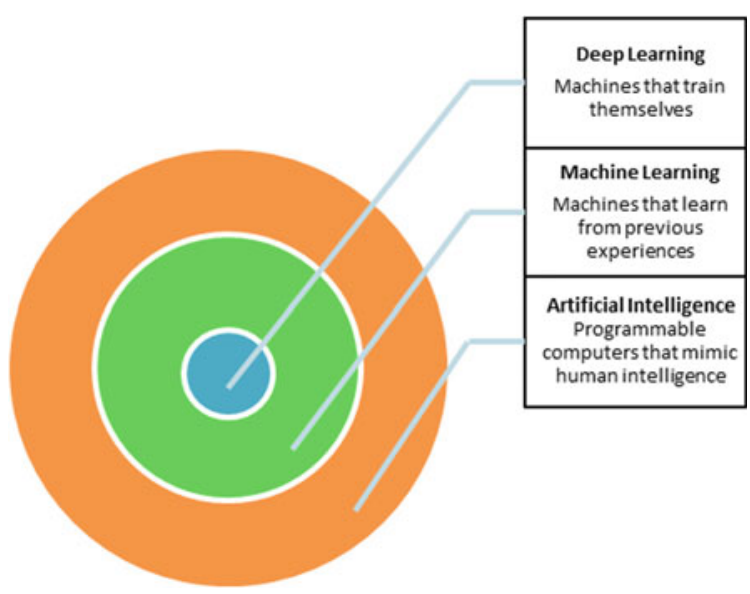

previous relevant data, learn from this data and predicts new outcomes [20]. ML applications are sub-divided into three categories:

I. Supervised learning: uses patterns of identified data (e.g. training data)

II. Unsupervised learning: finds and learns from patterns of data (e.g. data-mining that involves identification of patterns in large datasets)

III. Reinforcement learning: an extension of supervised learning that "rewards" and "punishes" when an application interacts with the environment.

Table 1 illustrates some common examples of supervised and unsupervised ML methods that are currently adopted and utilized to track health seeking behaviors during infectious disease epidemics [5].

Deep Learning (DL). DL is a specific subset of ML that uses neural networks (Fig. 4). In short, it is basically a synthetic replica of the human brain structure and functionality [4]. DL can execute multiple functions like image recognition and natural language processing (NLP). The system is capable of handling large datasets of information flow.

Image Recognition. AI has the capability to process large amount of data about characteristics of a particular phenomenon in the form of images or signals [20]. Motion images and sounds are examples of signals that could be analyzed using artificial neural networks (ANNs) [20]. Recently, researchers from the USA proposed a system that could rapidly identify potential arbovirus outbreaks (mosquito, ticks or other arthropod borne viruses) [21]. The system identifies images of mosquito larvae captured and delivered by a group of citizen scientists. Not only did the developed prototype facilitate collection of images, it also facilitated training of image classifiers for the recognition of a particular specimen. This sets a base for execution of expert validation process and data analytics. It was found that recognition of specimen in images provided by citizen scientists was useful to generate visualizations of susceptible geographical regions of arboviruses threat (Fig. 5). The system was capable of identifying mosquito larvae with great accuracy. 
Table 1 Common ML methods applicable to track health-behaviors during infectious disease epidemics

\begin{tabular}{l|l|l}
\hline Types & $\begin{array}{l}\text { Examples of } \\
\text { methods used }\end{array}$ & Functions \\
\hline $\begin{array}{l}\text { Supervised } \\
\text { method }\end{array}$ & $\begin{array}{l}\text { Support Vector } \\
\text { Machine (SVM), } \\
\text { Decision Tree, } \\
\text { Random Forest, } \\
\text { Naive Bayes (NB), } \\
\text { Artificial Neural } \\
\text { Network (ANN), } \\
\text { Bootstrap } \\
\text { Aggregating, } \\
\text { AdaBoost }\end{array}$ & $\begin{array}{l}\text { The primary function of these methods is to create } \\
\text { prediction outcomes to warn authorities and public in } \\
\text { advance on potential infectious disease epidemics, } \\
\text { thus suggesting immediate prevention and control } \\
\text { strategies }\end{array}$ \\
\hline $\begin{array}{l}\text { Unsupervised } \\
\text { method }\end{array}$ & $\begin{array}{l}\text { Principal Component } \\
\text { Analysis (PCA) }\end{array}$ & $\begin{array}{l}\text { This method aims to reduce data dimensions to enable } \\
\text { researchers easily uncover key factors causing } \\
\text { infectious disease dynamics }\end{array}$ \\
\cline { 2 - 4 } & K-Means & $\begin{array}{l}\text { This method clusters patients for abnormality } \\
\text { detections }\end{array}$ \\
\cline { 2 - 4 } & $\begin{array}{l}\text { Latent Dirichlet } \\
\text { Allocation (LDA) }\end{array}$ & $\begin{array}{l}\text { This method enables data extraction from medical } \\
\text { contextual records }\end{array}$ \\
\cline { 2 - 4 } & $\begin{array}{l}\text { Deep Learning } \\
\text { Architectures }\end{array}$ & $\begin{array}{l}\text { This method facilitates prediction and classification, } \\
\text { social network filtering and applications of } \\
\text { bioinformatics in infectious disease analytics }\end{array}$ \\
\cline { 2 - 4 } & $\begin{array}{l}\text { Structural Equation } \\
\text { Model Trees }\end{array}$ & $\begin{array}{l}\text { This method allows estimation of complex } \\
\text { cause-effect relationship models with latent variables }\end{array}$ \\
\hline
\end{tabular}

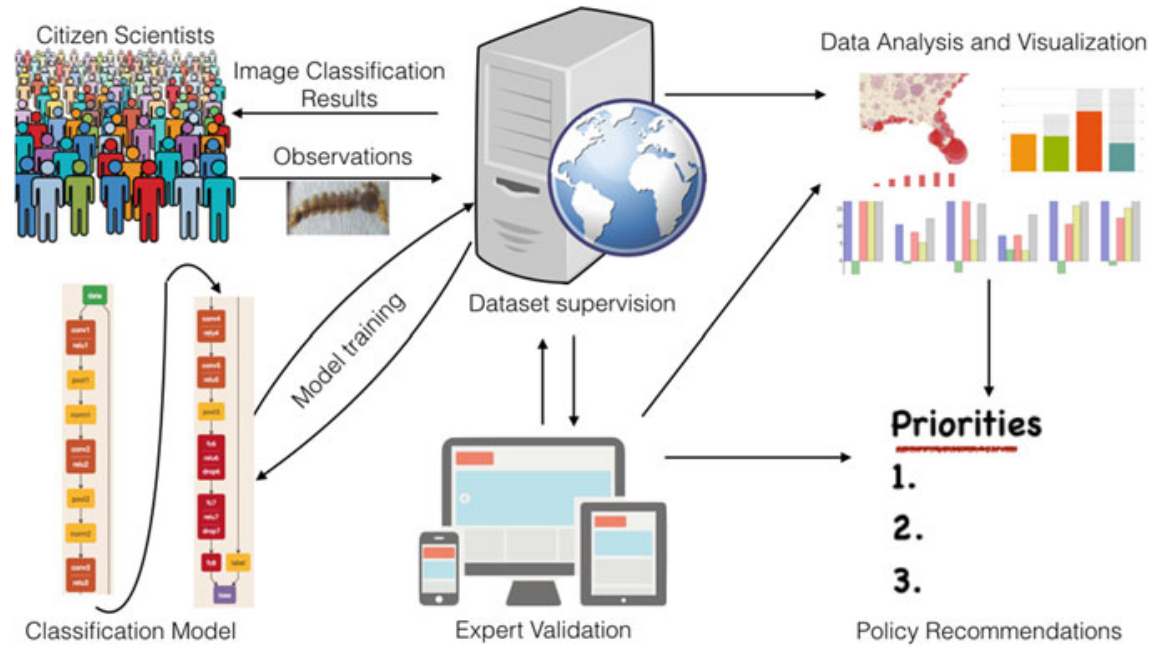

Fig. 5 Proposed system for rapid identification of arbovirus outbreaks. Source Munoz et al. [21] 
The rapid identification of potential outbreak to a susceptible community could alert preventive behaviors and policy drafting in the quest to control potential epidemics.

Natural Language Processing (NLP). NLP bridges the gap between languages that humans and machines use to operate. Algorithms are built to allow machines to identify keywords and phrases in an unstructured written text. AI applications then interpret the meaning of these texts for actionable knowledge [20].

Expert Systems (ES). ES incorporates expert-level competence to resolve a particular problem [20]. The system is constituted of two main components, namely knowledge base and a reasoning engine. It solves complex problems through reasoning a set of incomplete or uncertain information through a series of complex rules. In recent years, fuzzy logic, a set of mathematical principles for knowledge representation was crafted to accelerate the evolution of ES. Such strategy was utilized by a team of researchers from South Africa to improve predictions of cholera outbreaks [22].

\section{Applications of AI During Disease Epidemics: Concept Examples}

Public reaction and behavior towards disease outbreaks could be difficult to predict. With the rise of Big Data Analytics and a pool of AI applications in place, public health researchers were able to correlate population's behavior during an outbreak [5]. The following examples illustrate real life applications of AI during disease epidemics:

\subsection{The Use of Twitter Data to Track Public Behavior During Outbreak Crisis}

Twitter, a free social-networking micro-blogging service has enabled loads of users to send and read each other's "tweets (short, 140-character messages)." As important information and geo-political events are embedded within the Twitter stream, researchers now postulate that Twitter users' reactions may be useful for tracking and forecasting behavior during disease epidemics.

The Zika Pandemic. Most of the world's populations are living in endemic areas for common mosquito-borne diseases. The Zika pandemic between the years of 2015 and 2016 marked the largest known outbreak, reaching a "red-alert" warning of multiple complications requiring global public health interventions. In such exigencies, population health behaviors are important for potential control measures. Daughton and Paul postulated that internet data has been effective to track human health seeking behaviors during disease outbreaks [12]. They used 
Twitter data between 2015 and 2016 respectively to identify and describe self-disclosures of an individual's behavior change during disease spread. They combined keyword filtering and ML classifications to identify first-person reactions to Zika. A total of 29,386 English tweets were analyzed. Keywords include "travel," "travelling intentions," and "cancellations." Individual demographic characteristics, users' networking and linguistic patterns were compared with controls. The study found variations between individual characteristics, users' social network attributes and language styles in 1567 Twitter users. These users changed or considered to change their travel behaviors in response to Zika. Significant differences were observed between geographic areas in the USA, with higher discussion among women than men and some differences in levels of exposure to Zika-related information. This finding concludes that applying AI concepts could contribute to better understanding on how public perceives and reacts towards the risks of infectious disease outbreak.

The Influenza A H1N1 Pandemic. Signorini and colleagues in 2011 analyzed Twitter embedded data for tracking rapid evolvement of public concerns with respect to $\mathrm{H} 1 \mathrm{~N} 1$ or swine flu, while concurrently measuring actual disease activity [13]. The researchers explored public concerns by collecting tweets using pre-specified search terms related to $\mathrm{H} 1 \mathrm{~N} 1$ activity with additional keywords related to disease transmission, disease countermeasures and food consumption within the United States. They utilized influenza-like illness surveillance data and predicted an estimation model using supervised learning method in machine learning. The results showed that Twitter was useful to measure public interest or concern about health-related events associated with H1N1. These include an observed periodical spikes related to user Twitter activity that were linked to preventive measures (hand-hygiene practices and usage of masks), travel and food consumption behaviors, drug related tweets about specific anti-viral and vaccine uptake. They concluded that Twitter accurately estimated influenza outbreak through AI applications [13].

\subsection{Infodemiology and Infoveillance Studies to Track Health-Behaviors}

The integration of internet data into public health informatics has been regarded as a powerful tool to explore real-time human health-seeking behaviors during disease epidemics. One such popular tool widely utilized is Google Trends, an open tool that provides traffic information regarding trends, patterns and variations of online interests using user-specified keywords and topics over time [11]. Such adaptations formed two conceptualizations: the first was "Infodemiology," defined as "the science of distribution and determinants of information in an electronic medium, specifically the Internet, or in a population, with the ultimate aim to inform public 
health and public policy [23];" the second was "Infoveillance," defined as "the longitudinal tracking of infodemiology metrics for surveillance and trend analysis [24]."

Examining Health-Behavior Patterns during Dengue Outbreaks. Dengue is highly endemic across the South-East Asian countries. Recently, a group of researchers from the Philippines conducted an infodemiology and infoveillance study by using spatio-temporal concepts to explore relationships of weekly Google Dengue Trends (GDT) data from the internet and dengue incidence data from Manila city between 2009 and 2014 [25]. They subsequently examined health-seeking behaviors using dengue-related search queries from the population. Their findings suggested that weekly temporal GDT patterns were nearly similar to weekly dengue incidence reports. Themes retrieved from dengue-related search queries include: "dengue," "symptoms and signs of dengue," "treatment and prevention of dengue," "mosquito," and "other diseases." Most search queries were directed towards manifestations of dengue. The researchers concluded that GDT is a useful component to complement conventional disease surveillance methods. This concept could assists towards identifying dengue hotspots to facilitate appropriate and timely public health decisions and preventive strategies [25].

Health-Seeking Behavior of Ebola Outbreak. An unprecedented Ebola contagion that plagued most West African countries in 2014 marked the rise of global public health interest in pandemic preparedness interventions. Millions of Ebola-related internet hit searches were retrieved. With such high fluxes of health-seeking behavior using computers, a group of Italian researchers' evaluated Google Trends search queries for terms related to "Ebola" outbreak at the global level and across countries where primary cases of Ebola were reported [26]. The researchers subsequently explored correlations between overall and weekly web hit searches of terms in relation to the total number and weekly new cases of Ebola incidence. The highest search volumes that generated Ebola related queries were captured across the West African countries, mainly affected by the Ebola epidemic. Web searches were concentrated across state capitals. However, in Western countries, the distribution of web searches remained fixed across national territories. Correlations between the total number of new weekly cases of Ebola and the weekly Google Trends index varied from weak to moderate among the African countries afflicted by Ebola. Correlations between the total number of Ebola cases registered in all countries and the Google Trends index was relatively high. The researchers concluded that Google Trends data strongly correlated with global epidemiological data. Global agencies could utilize such information to correctly identify outbreaks, and craft appropriate actionable interventions for disease prevention urgently [26].

Public Reactions toward Chikungunya Outbreaks. The 2017 Italian outbreak of Chikungunya posed substantial public health concerns, catalyzing public interests in terms of internet searches and social media interactions. A group of researchers were determined to investigate Chikungunya-related digital health-seeking behaviors, and subsequently explored probable associations between epidemiological data and internet traffic sources [27]. Public reactions from Italy 
toward Chikungunya outbreaks were mined from Google Trends, Google News, Twitter traffics, Wikipedia visits and edits, and PubMed articles to yield a structural equation model. The relationships between overall Chikungunya cases, as well as autochthonous cases and tweet productions were mediated by Chikungunya-related web searches. But in the allochthonous case model, tweet productions were not significantly mediated by epidemiological figures, instead, web searches posed significant mediating tweets. Inconsistent associations were detected in mediation models involving Wikipedia usage. The effects between news consumption and tweets production were suppressed in this regard. Subsequently, inconsistent mediation effects were found between Wikipedia usage and tweets production, with web searches as a mediator. After adjustment of Internet Penetration Index, similar findings were retrieved with the adjusted model showing relationship between Google News and Twitter to be partially mediated by Wikipedia usage. The link between Wikipedia usage and PubMed/MEDLINE was fully mediated by Google News, and differed from the unadjusted model. The researchers found significant public reactions to the Chikungunya outbreak. They concluded that health authorities could be made aware immediately of such phenomenon with the aid of new technologies for collecting public concerns, disseminating awareness and avoiding misleading information [27].

\subsection{Computer Based Expert Systems}

Expert systems are built upon the basis to act as a diagnostic tool to accelerate detection of infectious disease epidemics, determining the intensity or concentration of vector-agents within the triads of infectious disease dynamics.

The Malaria Control Strategy Using Expert Systems. Malaria constitutes a "red-alert" health threat to the African communities. A group of researchers from Nigeria built an expert system for malaria environmental diagnosis with the aim of providing a decisional support tool for researchers and health policy-makers [28]. As prevailing malaria control measures were deemed insufficient, this group of researchers developed a prototype that constituted components of "knowledge," "applications," "system database," "user graphics interface," and "user components." The user component utilized Java, while the application component used Java Expert System Shell (JESS) and the Java IDE of Netbeans. The database component used SQL Server. The system was able to act as a diagnostic tool to determine the intensity of malarial parasites in designated geographical areas across Africa. The proposed prototype proved useful and cost-effective in curbing malaria spread [28]. 


\section{Challenges of AI Applications During Disease Epidemics}

Whereas $\mathrm{AI}$ is gaining increasing popularity and acceptance as a quick fix to the myriad of challenges faced with pandemic preparedness using traditional population-based approaches, it is not without its own limitations. Even in resource-rich settings, there are challenges associated with building and updating the knowledge base of expert systems [29], providing high-quality datasets upon which machine learning algorithms can be premised, and ethical issues associated with data ownership and management [20]. Additionally, resource-limited settings are further plagued with constraints of poorly organized and integrated health systems, poor IT and communication infrastructure, and socio-economic and cultural contexts [30, 31] that significantly impact successful implementation of AI systems. Beyond these, the dynamics of human behavior and other environmental covariates (such as mass/social media, public emotions, public policy etc.) may not only influence the accuracy of epidemic disease modeling frameworks but also impact health seeking behavior during epidemics [32]. More than ever before, public health experts, IT developers and other stakeholders must work together to address concerns related to scalability of AI for healthcare, data integration and interoperability, security, privacy and ethics of aggregated digital data. Finally, the transparency of predictive AI algorithms have been called to question, particularly given their 'black box' nature which makes them prone to biases in settings of significant inequalities [33].

\section{Opportunities and Future Direction of AI Applications During Disease Epidemics}

Perhaps, it may be premature to describe AI as the future of healthcare given it is still in its infancy, however, it has become increasingly difficult to not acknowledge the substantial contributions of AI systems to the field of public health medicine. Notwithstanding current challenges with the widespread adoption of AI particularly in resource-limited settings, the use of AI in providing in-depth knowledge on individuals' health, predicting population health risks and improving pandemic preparedness capabilities is likely to increase substantially in the near future [34]. Further, the rapidly expanding mobile phone penetrance, developments in cloud computing, substantial investments in health informatics, electronic medical records (EMRs) and mobile health (mHealth) applications, even in resource-constrained settings, holds significant promise for increasing use and scalability of AI applications in improving public health outcomes [35]. Public health policy, practice and research will continue to benefit from the expanding framework of infodemiology and infoveillance in analyzing health information search, communication and publication behavior on the internet [23, 24]. Advances in cryptographic technologies - including block chain is likely to allay fears and concerns with security, privacy and confidentiality of public digital data/information [36]. 


\section{Conclusion}

There is no doubt that AI is and will continue to revolutionize healthcare and population health. From prevention and health promotion to diagnosis and treatment, AI is increasingly being deployed to improve clinical decision-making, enhance personalized care and public health outcomes. In particular, AI offers enormous potential for cost-savings on therapeutic care given its predictive accuracy of potential outbreaks and epidemics and ability to enhance positive health seeking behaviors (at individual and population levels) during epidemics predicated upon robust infodemiology and infoveillance frameworks supported by expert systems, machine learning algorithms and mobile applications. Amazing as the future of AI in healthcare seems, there are significant legal and ethical concerns that need to be addressed in order to pave way for robust implementation and scalability across a variety of socio-cultural, epidemiological, health system and political contexts.

Acknowledgements We thank the Ministry of Health Malaysia for the support to publish this chapter.

\section{References}

1. K.L. Tsui, Z.S.Y. Wong, D. Goldsman, M. Edesess, Tracking infectious disease spread for global pandemic containment. IEEE Intell. Syst. 28(6), 60-64 (2013)

2. D. Baud, D.J. Gubler, B. Schaub, M.C. Lanteri, D. Musso, An update on Zika virus infection. Lancet 390, 2099-2109 (2017)

3. I.R.F. da Silva, J.A. Frontera, A.M.B. de Filippis, O.J.M.D. Nascimento, RIO-GBS-ZIKV Research Group, Neurologic complications associated with the Zika virus in Brazilian adults. JAMA. Neurol. 74(10), 1190-1198 (2017)

4. B. Mesko, G. Hetenyi, Z. Gyorffy, Will artificial intelligence solve the human crisis in healthcare? BMC Health. Serv. Res. 18, 545 (2018)

5. Z.S.Y. Wong, J. Zhou, Q. Zhang, Artificial intelligence for infectious disease big data analytics. Infect. Dis. Health. 24, 44-48 (2019)

6. S. Michie, J. Thomas, M. Johnston, P.M. Aonghusa, J. Shawe-Taylor, M.P. Kelly, L.A. Deleris, A.N. Finnerty, M.M. Marques, E. Norris, A. O'Mara-Eves, R. West, The human behavior-change project: harnessing the power of artificial intelligence and machine learning for evidence synthesis and interpretation. Implement. Sci. 12, 121 (2017)

7. H. Kagermann, H. Johannes, H. Ariane, W. Wolfgang, Recommendations for Implementing the Strategic Initiative INDUSTRIE 4.0: Securing the Future of German Manufacturing Industry. Final Report of the Industrie 4.0 Working Group (Forschungsunion, Frankfurt, Germany, 2013)

8. AI for Good Global Summit, Geneva (2017) http://www.itu.int/en/ITU-T/AI/Pages/201706default.aspx

9. United Nations: Looking to Future UN to Consider How Artificial Intelligence Could Help Achieve Economic Growth and Reduce Inequalities, http://www.un.org/sustainabledevelopment/ blog/2017/10/looking-to-future-un-to-consider-how-artificial-intelligence-could-help-achieveeconomic-growth-and-reduce-inequalities/2017 
10. J. Ginsberg, M.H. Mohebbi, R.S. Patel, L. Brammer, M.S. Smolinski, L. Brilliant, Detecting influenza epidemics using search engine query data. Nature 457(7232), 1012e4 (2009)

11. A. Mavragani, G. Ochoa, Google Trends in infodemiology and infoveillance: methodology framework. JMIR Public Health Surveill. 5(2), e13439 (2019)

12. A.R. Daughton, M.J. Paul, Identifying protective health behaviors on Twitter: observational study of travel advisories and Zika virus. J. Med. Internet Res. 21(5), e13090 (2019)

13. A. Signorini, A.M. Segre, P.M. Polgreen, The use of Twitter to track levels of disease activity and public concern in the US during the influenza A H1N1 pandemic. PLoS ONE 6(5), e19467 (2011)

14. V. Gianfredi, N.L. Bragazzi, D. Nucci, M. Martini, R. Rosselli, L. Minelli, M. Moretti, Harnessing big data for communicable tropical and sub-tropical disorders: implications from a systematic review of the literature. Front. Public Health 6, 90 (2018)

15. Air Transport Statistics 2018. International Air Transport Association (IATA), http://www. iata.org/services/statistics/air-transport-stats/Pages/index.aspx

16. N.L. Bragazzi, V. Gianfredi, M. Villarini, R. Rosselli, A. Nasr, A. Hussein, M. Martini, M. Behzadifar, Vaccines meet big data: state-of-the-art and future prospects. From the classical 3Is ("isolate-inactivate-inject") Vaccinology 1.0 to Vaccinology 3.0, vaccinomics and beyond: a historical overview. Front. Public Health 6, 62 (2018)

17. J. Mossong, N. Hens, M. Jit, P. Beutels, K. Auranen, R. Mikolajczyk, M. Massari, S, Salmaso, G.S. Tomba, J. Wallinga, J. Heijne, M. Sadkowska-Todys, M. Rosinska, W. J. Edmunds, Social contacts and mixing patterns relevant to the spread of infectious diseases. PLoS Med. 5(3), e74 (2008)

18. D. da Silva Motta, R. Badaro, A. Santos, F. Kirchner, Chapter 7: Use of artificial intelligence on the control of vector-borne diseases, in Vectors and Vector-Borne Zoonotic Diseases, ed. by S. Savic (IntechOpen, United Kingdom, 2018). ISBN 978-1-78985-293-6

19. C.S. Malley, J.C. Kuylenstierna, H.W. Vallack, D.K. Henze, H. Blencowe, M.R. Ashmore, Preterm birth associated with maternal fine particulate matter exposure: a global, regional and national assessment. Environ. Int. 101, 173-182 (2017)

20. B. Wahl, A. Cossy-Gantner, S. Germann, N.R. Schwalbe, Artificial intelligence (AI) and global health: how can AI contribute to health in resource-poor settings? BMJ Glob. Health $\mathbf{3}$, e000798 (2018)

21. J.P. Munoz, R. Boger, S. Dexter, J. Li, R. Low, Image recognition of disease-carrying insects: a system for combating infectious diseases using image classification techniques and citizen science, in Proceedings of the 51st Hawaii International Conference on System Sciences (HICSS, 2018), pp. 2835-2844. ISBN 978-0-9981331-1-9

22. G. Fleming, M. Mvander, G. McFerren, Fuzzy expert systems and GIS for cholera health risk prediction in southern Africa. Environ. Model. Softw. 22, 442-448 (2007)

23. G. Eysenbach, Infodemiology and infoveillance: framework for an emerging set of public health informatics methods to analyze search, communication and publication behavior on the internet. J. Med. Internet Res. 11(1), e11 (2009)

24. G. Eysenbach, Infodemiology and infoveillance: tracking online health information and cyber-behavior for public health. Am. J. Prev. Med. 40(5), S154-S158 (2011)

25. H.T. Ho, T.M. Carvajal, J.R. Bautista, J.D.R. Capistrano, K.M. Viacrusis, L.F.T. Hernandez, K. Watanabe, Using Google Trends to examine the spatio-temporal incidence and behavioral patterns of dengue disease: a case study in metropolitan Manila, Philippines. Trop. Med. Infect. Dis. 3, 118 (2018)

26. C. Alicino, N.L. Bragazzi, V. Faccio, D. Amicizia, D. Panatto, R. Gasparini, G. Icardi, A. Orsi, Assessing Ebola-related web search behavior: insights and implications from an analytical study of Google Trends-based query volumes. Infect. Dis. Poverty 4, 54 (2015)

27. N. Mahroum, M. Adawi, K. Sharif, R. Waknin, H. Mahagna, B. Bisharat, M. Mahamid, A. Abu-Much, H. Amital, N.L. Bragazzi, A. Watad, Public reaction to Chikungunya outbreaks in Italy -insights from an extensive novel data streams-based structural equation modeling analysis. PLoS ONE 13(5), e0197337 (2018) 
28. O. Oluwagbemi, E. Adeoye, S. Fatumo, Building a computer-based expert system for malaria environmental diagnosis: an alternative malaria control strategy. Egypt. Comput. Sci. J. 33(1), 55-69 (2009)

29. A. Sheikhtaheri, F. Sadoughi, Z.H. Dehaghi, Developing and using expert systems and neural networks in medicine: a review on benefits and challenges. J. Med. Syst. 38, 110 (2014)

30. A. Caliskan, J.J. Bryson, A. Narayanan, Semantics derived automatically from language corpora contain human-like biases. Science 356, 183-186 (2017)

31. J.L.K. Angwin, S. Mattu, L. Kirchner, Machine Bias (ProPublica, 2016)

32. R. Moss, A.E. Zarebski, S.J. Carlson, J.M. McCaw, Accounting for healthcare-seeking behaviors and testing practices in real-time influenza forecasts. Trop. Med. Infect. 4(1), 12 (2019)

33. IEEE Symposium, Algorithmic Transparency via Quantitative Input Influence: Theory and Experiments with Learning Systems. Security and Privacy (SP) (IEEE, 2016)

34. A. Shaban-Nejad, M. Michalowski, D.L. Buckeridge, Health intelligence: how artificial intelligence transforms population and personalized health. NPJ Digit. Med. 1, 53 (2018)

35. S. Feng, K.A. Grepin, R. Chunara, Tracking health seeking behavior during an Ebola outbreak via mobile phones and SMS. NPJ Digit. Med. 1(1), 51 (2018)

36. K. Ganasegeran, S.A. Abdulrahman, Adopting m-Health in clinical practice: a boon or a bane?, in Telemedicine Technologies, ed. by H.D. Jude, V.E. Balas (Elsevier Academic Press, United States, 2019), pp. 31-41 